\title{
Lessons from the Outbreak of E-cigarette-associated Lipoid Pneumonia in North Carolina
}

\author{
An Expert Interview with Kevin Davidson
}

WakeMed Health \& Hospitals, Raleigh, NC, USA

DOI: https://doi.org/10.17925/USPRD.2019.4.1.8

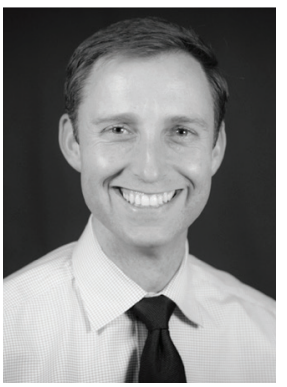

\section{Kevin Davidson}

Kevin Davidson MD is an interventional pulmonologist at WakeMed Hospitals in Raleigh, North Carolina. He completed his pulmonary and critical care fellowship at University of Colorado \& National Jewish Health in Denver, Colorado. He has completed subsequent subspecialty training in interventional pulmonology at Virginia Commonwealth University. He is the senior author of the Morbidity \& Mortality Weekly Report detalining the first cases of EVALI (e-cigarette- and vaping-associated lung injury) in North Carolina.

\section{Keywords}

E-cigarettes, acute lipoid pneumonia, acute lung injury, vaping

Disclosure: Kevin Davidson has nothihng to declare in relation to this article.

Review Process: This is an expert interview and, as such, has not undergone the journal's standard peer review process.

Compliance with Ethics: This article is an expert interview and did not involve any studies with human or animal subjects performed by any of the authors.

Authorship: The named author meets the International Committee of Medical Journal Editors (ICMJE) criteria for authorship of this manuscript, takes responsibility for the integrity of the work as a whole, and has given final approval for the version to be published.

Received: December 3, 2019

Published Online: January 27, 2020

Citation: US Respiratory \& Pulmonary Diseases.

2019;4(1):8-9

Corresponding Author: Kevin Davidson, WakeMed Health \& Hospitals, Raleigh, North Carolina, USA. E: kdavidson@wakemed.org

Support: No funding was received in the publication of this article.
$\mathrm{E}$ -cigarettes have rapidly grown in popularity across the US since they were introduced in 2007. The medical community has recently raised concerns about potential long-term health risks of e-cigarette use, and particularly about their popularity among young people. Kevin Davidson and his team recently reported cases of shortness of breath requiring oxygen, fevers, abnormal chest $\mathrm{x}$-rays, and chest computed tomography (CT) scans. These cases have recently been reported to the North Carolina Health Department and Centers for Disease Control (CDC).

\section{Q. What was previously known about the risks of e-cigarette use?} E-cigarettes gained rapid popularity in the US after initial release in 2007. Although they were initially marketed towards smokers as a cessation aid, their use became swiftly prevalent with younger audiences including teenagers and young adults who had never previously smoked. In that timeline, there were only rarely reported associations with alveolar hemorrhage, eosinophilic pneumonia, pneumonitis, and injury from explosions due to battery failures. The chief concerns from the medical community have been uncertainty regarding unknown long-term health risks of e-cigarette use and the increasing prevalence of nicotine dependence in our nation's youth.

\section{Q. Could you tell us a little about the e-cigarette-associated outbreak of acute lipoid pneumonia in North Carolina, including symptoms and clinical findings?}

In late July and early August, five young adults were admitted to our hospitals all with similar symptoms of respiratory failure. They had shortness of breath requiring oxygen, fevers, and abnormal chest $\mathrm{x}$-rays and chest CT scans, and often a prodrome of nausea, vomiting, or abdominal discomfort. Although there were initial concerns for possible pneumonia, they were not improving on antibiotics and diagnostic tests for other causes of infection were unrevealing. Our patients were quite ill and met criteria for acute respiratory distress syndrome (ARDS).

What linked all of these cases together was a shared history of vaping marijuana extracts and oils. Several of these patients underwent bronchoscopy with bronchoalveolar lavage and we recovered pulmonary macrophages with lipid-laden macrophages which stained positive for oil with specific lipid histologic stains. Although these findings are generally nonspecific and can be seen in a variety of conditions, our patients were otherwise healthy young patients without medical comorbidities. Based on their shared 
history of vaping marijuana oils and extracts, we knew they were inhaling atomized particles of these vaping liquids. We started these patients on corticosteroid therapy with concern for acute lipoid pneumonia and they subsequently improved, were able to come off of supplemental oxygen, and discharged home in excellent condition.

We immediately reported our findings and suspicions to the North Carolina Health Department and CDC. A report of our initial index cases was published in Morbidity and Mortality Weekly Reports to raise awareness of this outbreak. ${ }^{1}$ Our cases matched the symptoms and presentation that was characteristic of the national outbreak in the United States which peaked in the late summer 2019. Subsequently, even more of these cases have been identified and treated at our hospitals.

\section{Q. What potential explanations for this outbreak have been proposed?}

There has been extensive dialogue about what the particular culprit of e-cigarette- or vaping-associated lung injury (EVALI) may be. Our initial report raised concern that the mechanism may be an exogenous lipoid pneumonia linked to vaping of marijuana oils or extracts. Another group from the Mayo Clinic which reviewed lung biopsy specimens, raised concern that the culprit is a drug-induced lung injury. ${ }^{2}$ Subsequently, CDC laboratories have reported that vitamin $\mathrm{E}$ acetate has been identified as a chemical of concern within vaping products associated with the outbreak. ${ }^{3}$ Vitamin E, also known as tocopherol, is a cheap fat-soluble vitamin which is believed to added as an adulterant, or cutting agent, into some marijuana extracts or oils intended for vaping.

Interestingly, in early epidemiologic reports, we noted a disparity with fewer cases being reported in states such as Colorado and Washington where recreational marijuana is legal. However, more cases have been reported in states where recreational use of marijuana is illegal, and marijuana extracts and oils are sold on the street. Understandably, these illicit marijuana oils and extracts may pass through several transactions between their origin and the final user, with the possible addition of cutting agents at any step. The vast majority of all cases have been associated with vaping of marijuana and not nicotine. Even though many individual cases might smoke more than one substance, the associations of lung injury are far more prominent in those vaping marijuana.
Regardless of the precise pathophysiologic etiology, EVALI appears to be steroid-responsive unlike many other etiologies of ARDS. There may be several other factors which affect a patient's susceptibility to illness such as dose and duration of exposure, host-environment factors, or genetic factors which remain unknown.

\section{Q. What have we learned about the optimal treatment regimen and duration of this lung injury, and what are its likely long-term effects?}

only small retrospective reports have been published reporting that the majority of these cases are treated with corticosteroids and seem to improve. We typically treated our patients with 2 weeks of steroids but the optimal choice of steroid, dose, and duration are all unknown. The most important part of treatment and recovery is avoidance of smoking all substances including vaping marijuana or nicotine, smoking, or inhaling other illicit drugs. Our patients have been seen in follow-up with abnormal pulmonary function testing parameters which seem to improve within around 8 weeks. However, we are aware of more severe cases not seen at our center which did not improve and needed additional therapies such as extracorporeal membrane oxygenation or even lung transplantation.

\section{Q. What recommendations have been made on the basis of these findings?}

We strongly recommend for all healthcare providers to screen for e-cigarette/vaping use among patients, in particular those presenting with respiratory ailments. EVALI should be considered among other possible diagnoses and initiation of corticosteroids should be implemented early given a fitting clinical history, examination, laboratory evaluation, and chest imaging. In particular, we are concerned for vaping of marijuana extracts and oils because they have the strongest tie to the recent national outbreak of lung injury. We still encourage all of our patients to stop smoking both cigarettes and also nicotine or marijuana-containing e-cigarettes. We should remember that our experience with e-cigarettes is still relatively recent. The landmark United States Surgeon General's warning linking tobacco to lung cancer and heart disease wasn't issued until 1964; over half a century after cigarette smoking became commonplace. We still do not know the long-term risks associated with e-cigarette use and the recent outbreak of acute lung injury should be a reminder of the hazards of assuming that e-cigarettes and vaping are safe. $\square$

\footnotetext{
Davidson K, Brancato A, Heetderks P, et al. Outbreak of electronic-cigarette-associated acute lipoid pneumonia — North Carolina, July-August 2019. MMWR. 2019;68:784-6.

Butt Y, Smith M, Tazelaar H. Pathology of vaping-associated lung injury. N Eng/ J Med. 2019; 381:1780-1.

Blount B, Karwowski M, Shields P. Vitamin E acetate in bronchoalveolar-lavage fluid associated with EVALI. N Eng/ J Med. 2019. DOI: 10.1056/NEJMoa1916433. [Epub ahead of print].
} 